\title{
Structures of Self-Assembled $n$-Alkanethiols on Gold by Reflection High-Energy Electron Diffraction
}

\author{
Mithun Ghosh, Ding-Shyue Yang* \\ Department of Chemistry, University of Houston, Houston, Texas 77204, United States
}

*To whom correspondence should be addressed. Email: yang@uh.edu 


\begin{abstract}
The structures of long-chain alkanethiols $\left(\mathrm{C}_{18} \mathrm{H}_{37} \mathrm{SH}\right)$ chemisorbed on an $\mathrm{Au}(111)$ single crystal were investigated using reflection high-energy electron diffraction (RHEED). The primary $(\sqrt{3} \times \sqrt{3}) R 30^{\circ}$ structure observed as a major species in the as-deposited films contains gold adatoms below the sulfur headgroups. Between the small ordered domains with the alkyl chains tilting toward six directions are azimuthally disorderly packed regions, with a similar average tilt of $30.2^{\circ}$. In contrast, a significant reduction in the coverage of gold adatoms is found in the thermally-induced $(2 \sqrt{3} \times 3)_{\text {rect }}$ phase. This superlattice is shown to contain a mixture of two sulfur arrangements, both of which exhibit a small S-S distance, and the pairing of the aliphatic chains. A microscopic picture is then given for the structural transition. These findings demonstrate how the RHEED technique may be used to resolve structures of nanometer-thick thin films with multiple orders at the interfaces.
\end{abstract}

Keywords: self-assembled monoalyers, ODT SAM, thermal annealing, superlattice structure, adsorbate pairing 


\section{Introduction}

Since the discovery by Nuzzo and Allara in $1983,{ }^{1}$ self-assembled monolayers (SAMs) of organic thiols on gold have been utilized as an elegant yet versatile platform to better understand the physical and chemical phenomena involved in the self-assembly processes ${ }^{2-4}$ as well as their properties. ${ }^{5-9}$ The organized molecular architectures have also attracted broad attention as a result of their applications in various fields of nanotechnology and energy industries, e.g. chemical and biosensors, ${ }^{2,5,6}$ biotechnology ${ }^{3,4}$ and molecular electronic devices, ${ }^{7-9}$ solar cells, ${ }^{10}$ electrochemistry, ${ }^{11}$ and organic field-effect transistors. ${ }^{12}$ To better characterize the organizations of alkanethiols chemisorbed on gold, a plethora of surface science techniques have been used to provide the structural information either in a local region using atomic force microscopy ${ }^{13,14}$ and scanning tunneling microscopy (STM) ${ }^{15,16}$ or from a large probed area to capture an ensemble picture using surface-sensitive methods such as infrared spectroscopy, ${ }^{17,18}$ optical ellipsometry, ${ }^{18}$ X-ray photoelectron spectroscopy (XPS) ${ }^{19}$ helium atom scattering, ${ }^{20}$ low-energy electron diffraction (LEED), ${ }^{17}$ and grazing incidence X-ray diffraction (GIXD) ${ }^{21}$ Both types of methods are necessary to reach a more complete understanding. ${ }^{22,23}$

Early studies described a commensurate $(\sqrt{3} \times \sqrt{3}) R 30^{\circ}$ primary structure for chemisorbed long-chain ( 10 or more carbon atoms) $n$-alkanethiols at the saturation coverage relative to the underlying $\mathrm{Au}(111)$ substrate lattice (Figure 1a-b). ${ }^{24-26}$ The nearest-neighbor displacement between all-trans thiol molecules is $\sim 5.0 \AA$, leading to an area of $\sim 21.4 \AA^{2}$ as the calculated coverage per molecule. In addition, the alkyl chains are tilted towards the next-nearest-neighbor direction with an angle of approximately $30^{\circ}$ from the surface normal direction. ${ }^{24}$ However, later reports indicated the existence of a secondary ordering of thiols, first hinted by the splitting of the methylene scissor mode observed by infrared spectroscopy at low temperature ${ }^{27}$ and then 


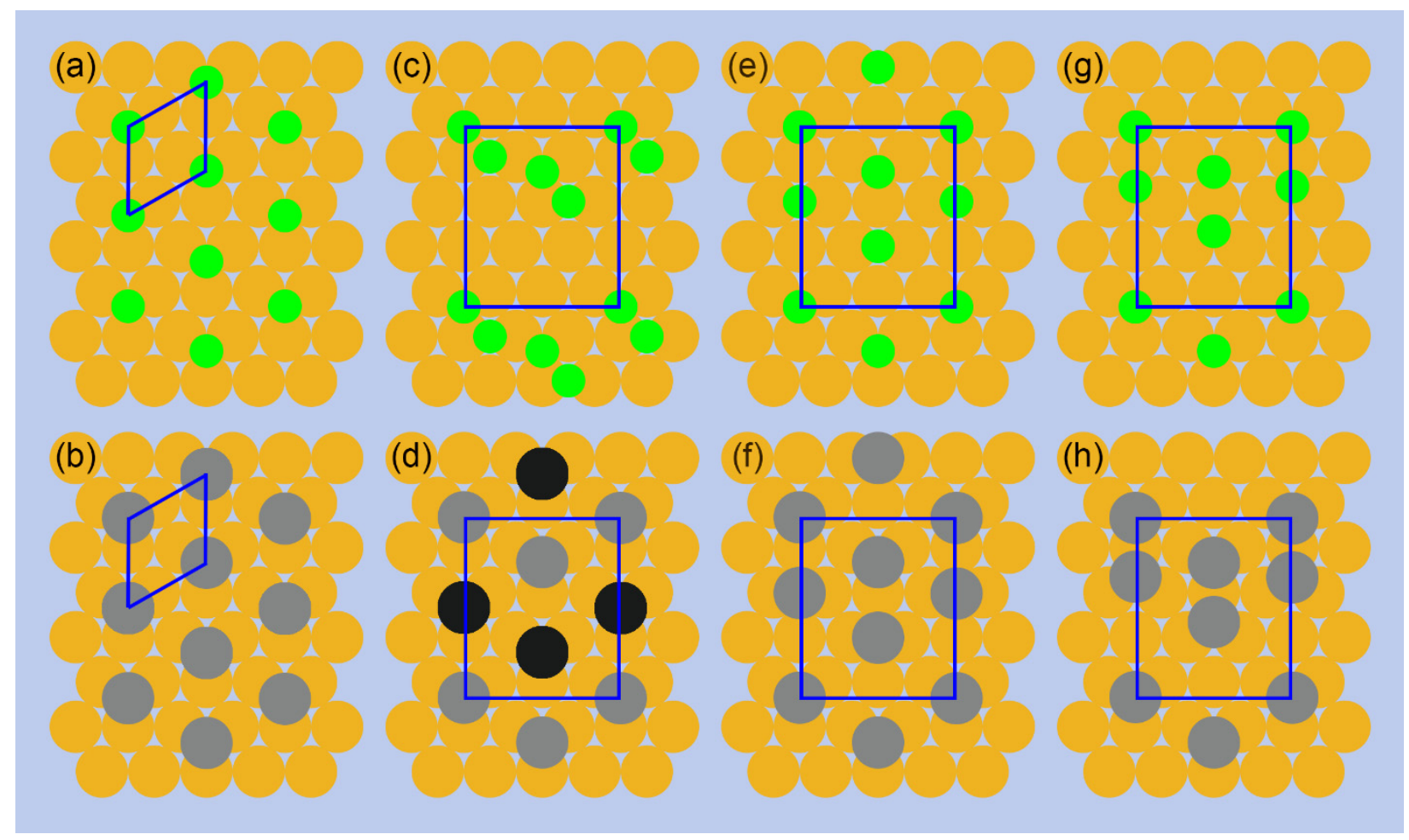

Figure 1. Different two-dimensional arrangements proposed in literature for the sulfur sublattice (top panels) and the tailgroups of aliphatic chains (bottom panels). (a, b) $(\sqrt{3} \times \sqrt{3}) R 30^{\circ}$ structures. (c) A S-S dimer model. (d) A twisted-chain model giving the $(2 \sqrt{3} \times 3)_{\text {rect }}$ superlattice. $(\mathrm{e}-\mathrm{h})$ Two sets of adsorbate-pairing models producing the $(2 \sqrt{3} \times 3)_{\text {rect }}$ superlattice. identified by diffraction ${ }^{20}$ and $\mathrm{STM}^{28}$ as a $c(4 \times 2)$ superlattice relative to the former $(\sqrt{3} \times$ $\sqrt{3}) R 30^{\circ}$ structure [or in a rectangular $(2 \sqrt{3} \times 3)_{\text {rect }}$ superlattice relative to $\mathrm{Au}(111)$ ]; some studies used shorter-chain ( 8 or fewer carbon atoms) alkanethiols. ${ }^{29,30}$ It has also been shown that a thermal treatment of long-chain SAMs on $\mathrm{Au}(111)$ results in the formation of larger ordered domains and facilitates the transition from the primary structure to the thermodynamically more stable $(2 \sqrt{3} \times 3)_{\text {rect }}$ phase. $^{31,32}$ However, despite the extensive efforts both experimentally and theoretically, which have led to various proposed models, ${ }^{23,24,33-36}$ the actual superlattice structure is still under debate (Figure 1). 
Fundamentally, the organizations of sulfur headgroups, methylene spacers, and tail functional groups are all needed to determine the structure of a SAM on gold. Here, we limit the discussion for assemblies of long-chain $n$-alkanethiols and hence are only concerned about two arrangements, i.e. that of the sulfur atoms and that of the remaining alkyl chains as a whole. Initially, a model containing two inequivalent molecules in a $(2 \sqrt{3} \times 3)_{\text {rect }}$ unit cell with different twist angles along the methylene backbones was proposed, considering the high sensitivity of helium atom scattering ${ }^{20}$ and $\mathrm{STM}^{29}$ toward the topmost part of an assembly (Figure 1d). Thus, the orientations of the alkyl chains were regarded as the key ingredient of the superlattice, whereas the sulfur arrangement was not directly resolved but assumed to bind the three-fold face-centered-cubic (fcc) interstitial sites of $\mathrm{Au}(111)$ (Figure 1a). Such a model was first called into question by Fenter et al., whose GIXD results signified the presence of two inequivalent sulfur headgroups forming S-S dimers (Figure 1c) along with different twist angles of the alkyl chains (Figure 1d) for the superlattice structure. ${ }^{21}$ Subsequently, a number of studies supported this dimer model with direct or indirect experimental observations using sum frequency generation, ${ }^{37}$ high-resolution XPS,${ }^{38}$ thermal desorption spectroscopy, ${ }^{39} \mathrm{STM},{ }^{40}$ and X-ray standing wave method, ${ }^{41}$ all of which have different sensitivities toward the sulfur sublattice. However, it was a high-resolution energy-loss spectroscopy (HREELS) study that provided direct evidence for the observation of disulfide bonds on $\mathrm{Au}(111)$ at $375 \mathrm{~K}$ but their absence in a freshly prepared sample. ${ }^{42}$ Interestingly, radiation-induced breakage of S-Au bonds and formation of disulfides were also cautioned in an earlier XPS study, which supported the presence of disulfide bonds at an elevated temperature. ${ }^{43}$

An intriguing turn of the story was seen following a later GIXD study by Torrelles et $a l .{ }^{44}$ which proposed a different sulfur sublattice against actual dimerization (Figure 1g). The 
distance between neighboring adsorbate atoms was indicated to be $3.2 \AA$, with the two sets of sulfur atoms occupying nearby fcc and hexagonal-closest-packed (hcp) interstitial sites. With a short butyl chain for the alkanethiolates, Chaudhuri et al. by symmetry arguments provided support for this fcc-hcp adsorption scheme as one of the possible structures that agree with the data by both LEED and GIXD (from long-chain thiols), although two other models with gold adatoms below sulfur atoms cannot be ruled out. ${ }^{36}$ However, it was also around a similar time that the latter gold adatom models gained more recognition, first pointed out by Maksymovych et al. on still relatively short hexanethiols and octanethiols using normal incidence X-ray standing waves. ${ }^{45}$ Details in a later STM observation of short-chain octanethiols led to the interpretation of the apparent pairing found in the images as support for thiolates binding directly on gold adatoms; the organization of the alkyl chains was not a central issue. ${ }^{30}$ Nonetheless, the effects of an STM tip on the structures of SAMs and image formation and interpretation have been the major challenges for this scanning probe microscopy method. ${ }^{30,31,46}$ It is intriguing that more than seven different $(2 \sqrt{3} \times 3)_{\text {rect }}$ structures have been proposed by STM studies, which are only a slight variant of each other, ${ }^{22,28,30}$ whereas relative few were presented in diffraction-based studies $^{20,21}$ as the most abundant structures. This raises a crucial question: what is the best model to describe the $(2 \sqrt{3} \times 3)_{\text {rect }}$ superlattice? Further investigation using a different surface sensitive technique is thus necessary. In addition, a clear understanding of the equilibrium structure is also critical to the interpretation of stimulus-induced dynamics and changes.

In this report, reflection high-energy electron diffraction (RHEED) is used to examine the structures of octadecanethiols (ODTs) chemisorbed on single-crystalline Au(111). The RHEED method has not been applied on SAMs to date, although its use has been seen in studies of Langmuir-Blodgett (LB) films of fatty acids to determine the packing of alkyl chains. ${ }^{47-49}$ 
Compared to X-ray photons, ${ }^{21,44}$ electrons have orders-of-magnitude higher scattering cross sections with matter, which readily enables the probing of different parts of a molecular assembly by changing the incidence angle. Furthermore, it is relatively straightforward to distinguish the contributors of various diffraction features, thus capable of resolving questions regarding the buried headgroup structure, its relation with that of the tailgroups, and additional features from the substrate. Determination of the molecular tilt of the alkyl chains from the substrate surface normal is also straightforward..$^{50}$

We first establish the effectiveness of the RHEED method by studying the as-prepared SAMs of ODTs, which exhibit a crystalline arrangement for the headgroups in a large area on $\mathrm{Au}(111)$ and a substantial degree of disorder in the alkyl chain region that forms 6-fold rotated small ordered domains coexisting with imperfect, randomly oriented molecular packing. However, the average molecular tilt is the same, $30.2^{\circ} \pm 0.4^{\circ}$. This predominantly hexagonal $(\sqrt{3} \times \sqrt{3}) R 30^{\circ}$ overlayer evolves to show a longer-range order for the methylene packing under thermal annealing. Furthermore, with clear increase of the average domain size, distinguished diffraction features from the sulfur atoms and the alkyl chains show the formation of the superlattice structures that involve different arrangements for the head and tail groups. More importantly, with the help of kinematic scattering simulations for diffraction, evidence is found for the presence of gold adatoms under sulfur in the as-prepared SAMs but not in the thermallytreated films. We believe that these RHEED observations of SAM ODTs are helpful to reconcile the different superlattice models proposed in the literature and thus provide information about the major contributing structures formed on $\mathrm{Au}(111){ }^{21,44}$

\section{Experimental}

A single-crystalline $\mathrm{Au}(111)$ substrate with a surface orientation accuracy of $<0.1^{\circ}$ (Princeton 
Scientific Corp.) was used to reach the best structural order of SAMs. The crystal was cleaned by repeated rounds of argon ion sputtering at $1.5 \mathrm{keV}$ at room temperature and annealing for hours at $770 \mathrm{~K}$ in vacuum at a base pressure of $<2 \times 10^{-8}$ torr. The atomically flat $\mathrm{Au}(111)$ surface was confirmed by the characteristic streaky RHEED pattern at grazing incidence angles without observation of any transmission-like diffraction spots. The gold substrate was then removed from vacuum and immediately used in the solution-based preparation of SAMs. ${ }^{51}$ In short, $\mathrm{Au}(111)$ was immersed in a 1-mM solution of ODT in ethanol for 46 hours in a nitrogen-filled glove box; octadecanethiol (98\%, from Sigma Aldrich) was used as received without further purification. The SAM samples were rinsed with a copious amount of ethanol and dried under ultrahigh pure helium and then loaded into vacuum. The time of ambient exposure during sample rinsing, drying, transport, and loading was no more than 30 minutes. The sample plate was mounted on a 5-axis goniometer coupled to a cryostat for liquid nitrogen cooling and internal heating up to 460 $\mathrm{K}$. The surface temperature was measured directly by a K-type thermocouple with an accuracy of $<1 \mathrm{~K}$. In the present study, samples were annealed up to a range of 373-383 K. Diffraction experiments were conducted with $30-\mathrm{keV}$ electrons by varying the grazing incidence angle $\left(\theta_{\text {in }}\right)$ between $0^{\circ}$ and $5^{\circ}$ and the accessible range of the azimuthal angle $(\phi)$. A schematic of the RHEED geometry is shown in Fig. S1. The resulting RHEED images on a phosphor screen were recorded by an intensified CMOS camera assembly, which allows the data acquisition using an ultralow rate of electron dose on the order of $0.1-1 \mathrm{nA} / \mathrm{cm}^{2}$. The fabrication of SAMs and the RHEED experiments were conducted and reproduced multiple times. No changes were observed in the diffraction patterns even after extended exposure to the electron beam (still a low dose of $0.001-0.01 e / \AA^{2}$ for 10 consecutive hours). Thus, we confirm that radiation damage of SAMs is of minimal concern in our study. 


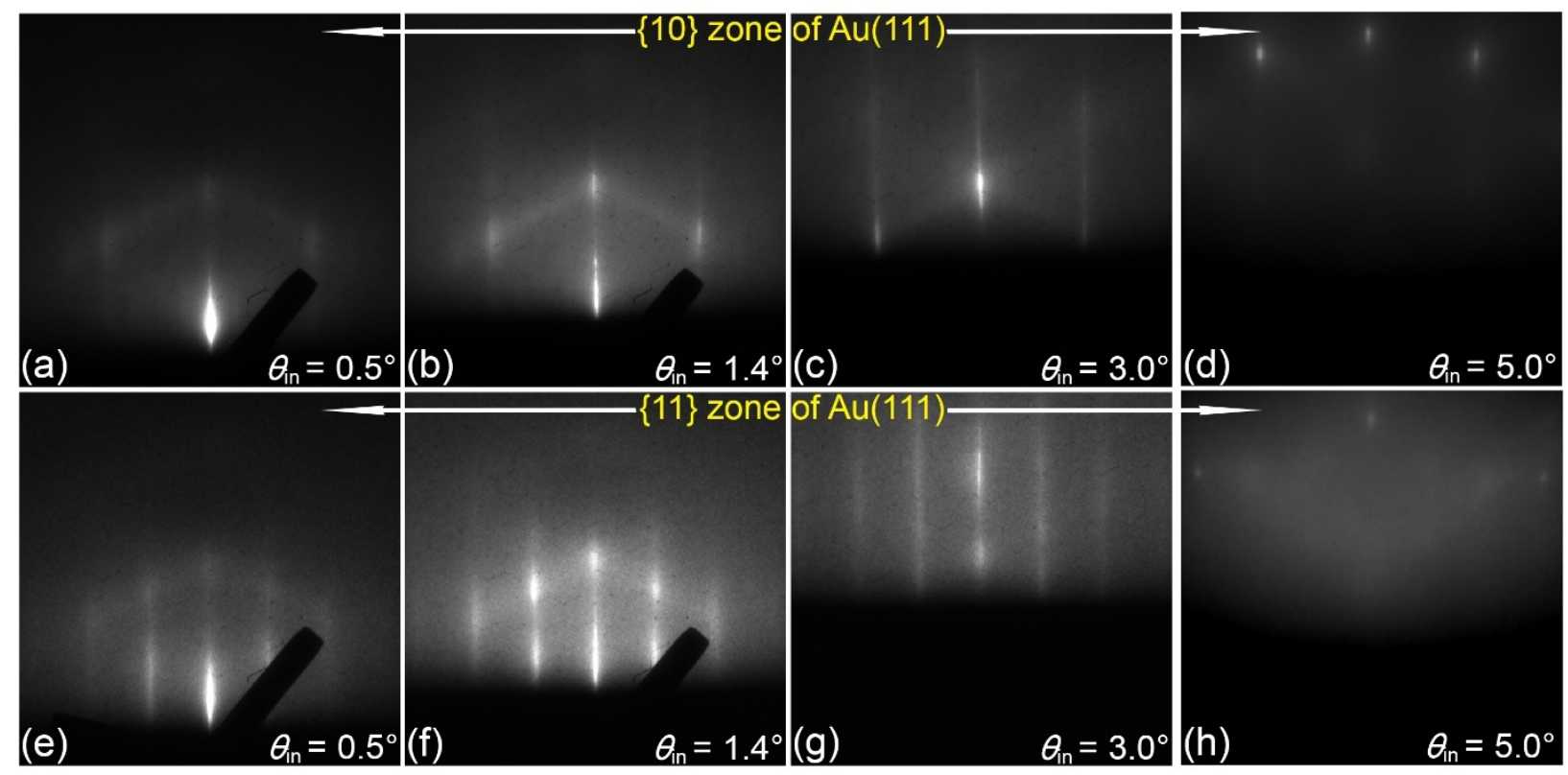

Figure 2. RHEED images acquired at selected incidence angles from an as-deposited specimen. Top (bottom) panels are recorded with electrons probing the $\{10\}(\{11\})$ zones of $\mathrm{Au}(111)$; the images in panels $d$ and $h$ are displaced vertically in order to show the diffraction spots from the supporting gold substrate. The hut-shaped diffraction feature is observed at lower incidence angles. Note the different horizontal spacings between panels e-g and h.

\section{Results \& discussion}

\subsection{Primary structure}

The repeating $\mathrm{C}_{2} \mathrm{H}_{4}$ methylene units in an ordered SAM of ODTs are anticipated to form a threedimensional (3D) lattice for ordered domains, which should resemble what was previously observed in LB films of long-chain fatty acids. ${ }^{47,48}$ With an estimated packing density of the aliphatic chains $(N)$ and the total elastic scattering cross section of $30-\mathrm{keV}$ electrons by carbon and hydrogen atoms $(\sigma)$, a mean free path of $l=1 / N \sigma \sim 130 \mathrm{~nm}$ is obtained. Therefore, the diffraction probe depth of $\sim 130 \mathrm{~nm} \cdot \sin \theta_{\text {in }}$ is estimated and essentially only the $\mathrm{C}_{2} \mathrm{H}_{4}$ sublattice of the SAM is probed when $\theta_{\mathrm{in}} \leq 1^{\circ}$ for a film thickness of $\sim 2 \mathrm{~nm}$. The sulfur headgroup 
sublattice and the crystalline gold substrate are more accessed at higher $\theta_{\text {in }}$ because of the increased probe depth.

Our observations are consistent with this simple estimate. Shown in Figure 2 are the RHEED images of an as-prepared SAM at selected $\theta_{\text {in }}$ 's, with the electron path directed along two principal crystallographic directions $\left(30^{\circ}\right.$ apart) of $\mathrm{Au}(111)$. Four main diffraction features can be distinguished. First, a somewhat diffuse hut-shaped feature symmetric with respect to the center vertical rod is recognizable at all $\theta_{\text {in }} \leq 3^{\circ}$ and invariant to the probing direction [i.e., $\phi ; 0$ degree is defined to match with probing a (10) zone of gold] (Figure 2, a-c and e-g). Second, spotty diffractions, either well-defined or vertically elongated, are found prominent at lower $\theta_{\text {in }}$ and become symmetric in their appearance at selected $\phi^{\prime}$ s, particularly along the two principal zones of $\mathrm{Au}(111)$ (Figure 2, a, b, e, and f). Such a feature signifies the presence of a 3D lattice in the SAM with respect to the substrate. Third, more streak-like diffractions appear when $\theta_{\text {in }}>1^{\circ}$ and are more prominent at higher $\theta_{\mathrm{in}}$, which agrees with the probing of a different 2D lattice in the lower region of the SAM and the top surface of $\mathrm{Au}(111)$ (Figure 2, b, c, f, and g). The coincidence in the horizontal spacing and arrangement of the former Bragg spots and these diffraction streaks is noted, which indicates the relation of the azimuthal orders between the 3D methylene and the $2 \mathrm{D}$ sulfur sublattices. At $\theta_{\text {in }}>4.5^{\circ}$, the diffraction spots from $\mathrm{Au}(111)$ can be seen (Figure 2, $d$ and $h$ ), showing more penetration of the probe electrons through the SAM to detect the surface of the substrate.

In the present case, it is relatively straightforward to use the crystallographic concepts of reciprocal rods and the Ewald construction to find out the structural relation between the $2 \mathrm{D}$ sulfur sublattice and $\mathrm{Au}(111)$. Their hexagonal structures are confirmed by the recurring patterns in a $\phi$ scan, indicating a 6-fold rotational symmetry. The smallest horizontal spacings observed 
between diffraction rods are directly associated with probing of the $\{10\}$ zones (Figure $2 \mathrm{~d}$ for the gold substrate and Figure $2 \mathrm{f}-\mathrm{g}$ for sulfur). This association is also confirmed by the observation of $\sqrt{3}$ times the former spacings in the patterns acquired at $30^{\circ}$ azimuthal rotation where the $\{11\}$ zones are probed (Figure $2 \mathrm{~h}$ for gold and Figure $2 \mathrm{~b}-\mathrm{c}$ for sulfur). Hence, the unit-cell constant $a$ for a hexagonal lattice can be calculated using the following equation,

$$
a=\frac{2 \pi}{s \cdot \sin 60^{\circ}}
$$

where $s$ is the momentum transfer associated with the smallest horizontal spacing. From the known $a_{\mathrm{Au}}=2.884 \AA$ for $\mathrm{Au}(111), a_{\mathrm{S}}=5.00 \pm 0.02 \AA$ is obtained, which is $\sqrt{3} \cdot a_{\mathrm{Au}}$. Thus, combining with the fact that the $\{10\}$ zone of the sulfur lattice and that of $\mathrm{Au}(111)$ are separated by $30^{\circ}$, we establish that the sulfur headgroups indeed form a $(\sqrt{3} \times \sqrt{3}) R 30^{\circ}$ hexagonal overlayer in the primary structure with respect to the gold lattice, which is consistent with the literature (Figure 1a).

Packing of the 3D methylene lattice is hence expected to be hexagonal as well given the covalent bonding with the sulfur atoms. The tilt angle (with respect to the surface normal) and the azimuthal orientation (with respect to the substrate) of the aliphatic chains need to be determined; results from previous studies of LB films may be used as a reference for the intracell atomic arrangement. The angular information can be deduced based on the relative positions of the Bragg spots observed at low $\theta_{\text {in }}$ and the angles of the hut-shaped diffraction feature with respect to the shadow edge (Figure 2, a, b, e, and f; see also the discussion below regarding Azimuthally Disordered Domains). To confirm, we carry out simulations of RHEED patterns from an ordered monolayer of ODTs using the kinematic scattering theory. The intensity $I_{h k l}$ of a Bragg spot $(h k l)$ is proportional to the square of the structure factor $F_{h k l}$, 


$$
F_{h k l}=\sum_{j} f_{j} \exp \left[2 \pi i\left(h x_{j}+k y_{j}+l z_{j}\right)\right]
$$

where the sum is calculated over all atoms in the unit cell with $\left(x_{j}, y_{j}, z_{j}\right)$ being the coordinates of the $j$ th atom and $f_{j}$ the corresponding scattering factor.

The following main observations are found as a result. First, the ordered domains of the methylene lattice, although forming a hexagonal arrangement (Figure 1b), do not all exhibit the same azimuthal orientation for the molecular tilt even though a defined value for the tilt angle is

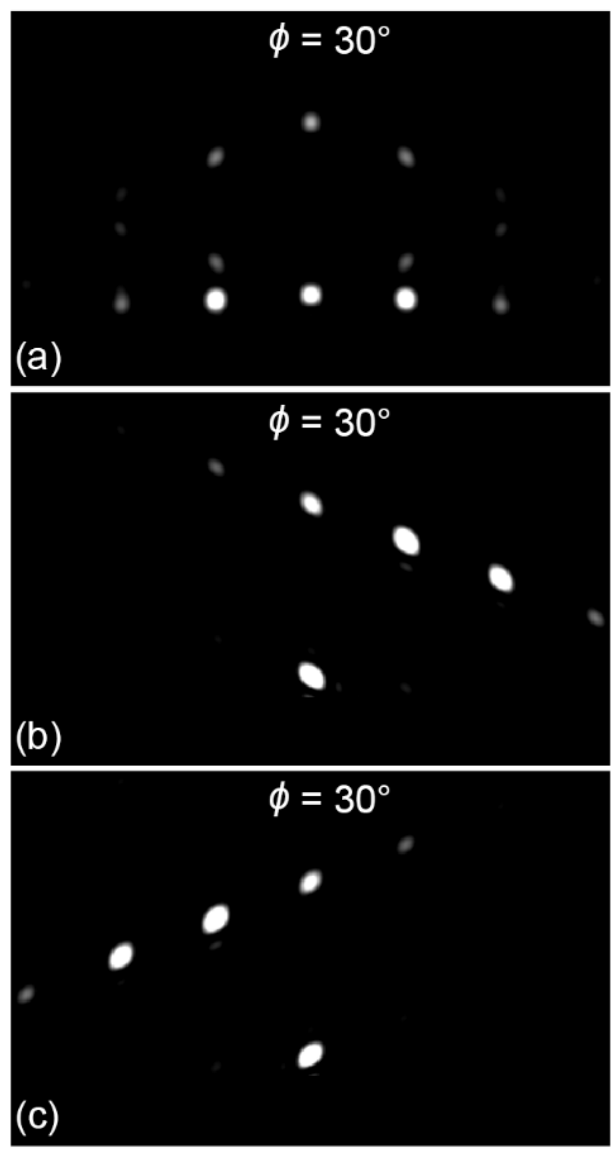

Figure 3. Simulated RHEED patterns generated using different azimuthally rotated domains of the $(\sqrt{3} \times \sqrt{3}) R 30^{\circ}$ structure of the ODT SAM at $\phi=30^{\circ}$. (a) The accumulated pattern obtained from all six-fold rotated domains. $(b, c)$ A slanted pattern from the domain where the tilt direction of the molecules is $+90^{\circ}\left(-90^{\circ}\right)$ with respect to the electron probe direction. 
obtained. In fact, the symmetric spot patterns observed in Figure 2, b and f, along both principal axes of $\mathrm{Au}(111)$ indicate a uniform presence of all six azimuthally-rotated domains (hence each with one of the 6-fold tilt directions) of the alkyl chains in the probed region (Figure 3). Second, the width of the Bragg spots gives $\sim 9.3 \mathrm{~nm}$ as the average diameter of the crystalline domains according to the Scherrer formula. ${ }^{52}$ Thus, these two results show that the common SAM growth
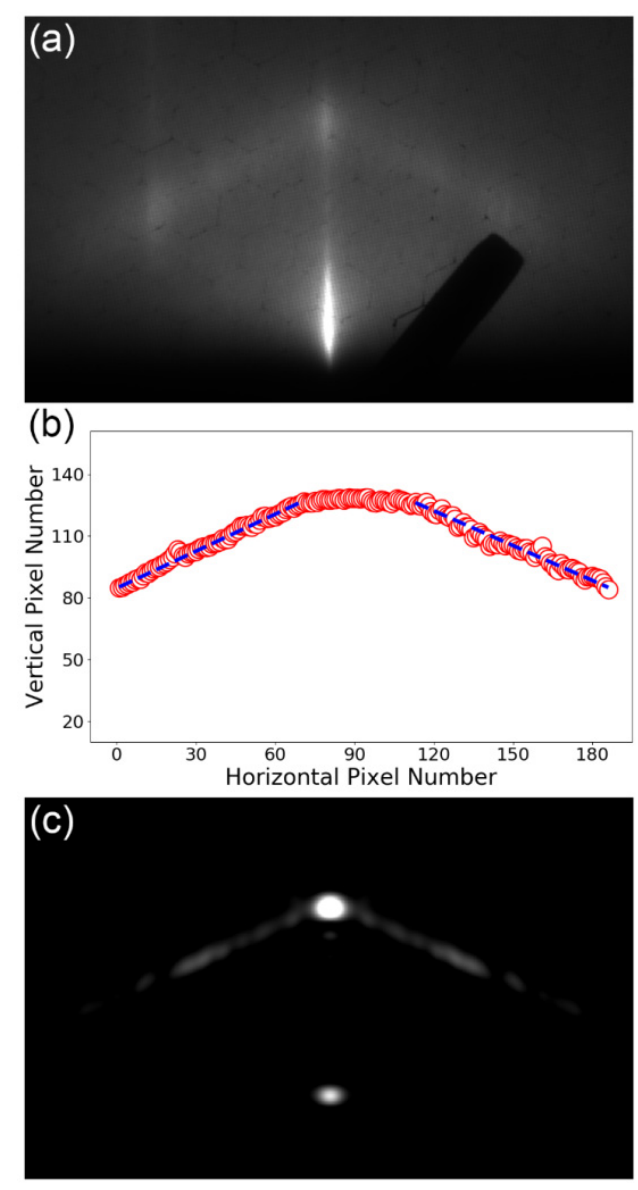

Figure 4. The hut-shaped diffraction feature. (a) An experimental RHEED image obtained from an as-deposited specimen by averaging over a $\phi$ range of $25^{\circ}$. (b) The vertical center position of the hut-shaped feature obtained at each horizontal pixel with a fit of the intensity profile to a Gaussian function over a quadratic background. The dashed lines are linear fits. (c) Simulated hut-shaped pattern obtained from azimuthally rotated domains with a random distribution of the lattice constant around a mean value of $5.0 \AA$. 
at room temperature only results in a limited size of ordered methylene packing [above the more regular $(\sqrt{3} \times \sqrt{3}) R 30^{\circ}$ headgroups], even after an extended incubation period. This is consistent with results of STM studies showing the presence of the six-fold rotational $(\sqrt{3} \times \sqrt{3}) R 30^{\circ}$ domains with the tilting of the alkyl chains along one of the six next-nearest-neighbor directions. ${ }^{40,53}$ Additionally, it is found that the sulfur atoms alone would not have sufficient contribution to the intensity of the diffraction streaks. Further discussion about this point will be made later.

\subsection{Azimuthally disordered domains}

Locally packed aliphatic chains are anticipated to be present in the gaps between the 6-fold ordered domains. This is supported by the aforementioned hut-shaped diffuse feature, which does not change for all $\phi$ probed and hence further indicates the alkanes to be imperfectly

ordered (see below) ${ }^{50}$ However, the inclination of the two diffuse wings referenced to the shadow edge still gives a good measure of the average chain tilt with respect to the surface normal. Shown in Figure 4a is an average RHEED image over a $\phi$ range of $25^{\circ}$. The center of the diffraction wings at each horizontal image pixel is then determined by a fit of the vertical intensity profile to a Gaussian function over a quadratic background (Figure $4 \mathrm{~b}$ ). With linear fits, a value of $30.2^{\circ} \pm 0.4^{\circ}$ is determined for the average molecular tilt, which is within the range reported previously in the literature..$^{22,54}$

Shown in Figure $4 \mathrm{c}$ is a simulated RHEED pattern that resembles the experimental hutshaped observation in Figure 4a. Here, a uniform molecular tilt of $30^{\circ}$ is used but the horizontal displacement between adjacent molecules is randomly altered and given a small distribution around $5.0 \AA$; the simulated results are then aggregated azimuthally over $360^{\circ}$ rotation. Thus, 
this diffraction result further signifies that, in addition to the inherent distribution of the tilt angle, a translational disorder from the hexagonal packing exists among the azimuthally disordered aliphatic chains that fill up between the 6-fold ordered crystalline domains. We also note that the diffraction spots are on the hut-shaped feature (Figure 2, a, b, e, and f), which indicates the similar chain tilt for both ordered and disordered regions. Consequently, a picture of mixed orders and orientations of $\sim 30^{\circ}$-tilted alkyl packing with the sulfur headgroups forming a $(\sqrt{3} \times \sqrt{3}) R 30^{\circ}$ overlayer is concluded for as-prepared SAMs of long-chain thiols on $\mathrm{Au}(111)$ at room temperature, which is in good agreement with the generally accepted picture of SAMs. The azimuthally disordered regions are referred as "liquid-like domains" in STM studies. ${ }^{22,32}$ Also, fluctuations of the lattice spacings were previously observed based on the GIXD data. ${ }^{55}$

The hut-shaped feature becomes weaker as the SAM is annealed at elevated temperatures and almost disappears at $383 \mathrm{~K}$ (Figures 5 and 6 , recorded at $\sim 100 \mathrm{~K}$ ). This result suggests that a high-temperature thermal treatment helps to further enlarge those ordered domains (to an average size of $\sim 87 \mathrm{~nm}$ estimated using the Scherrer formula) and therefore reduce the disordered regions in between. However, new diffraction features different from what are seen in Figure 2 also emerge, which indicate the growth of (and the conversion to) a secondary structure in the SAM.

\section{$3.3(2 \sqrt{3} \times 3)_{\text {rect }}$ Superlattice structure}

Shown in Figure 5 are the experimental and simulated RHEED images at two selected incidence angles to highlight the new diffractions from the chain and headgroup sublattices after a specimen was annealed for 5 minutes. A quick comparison of Figure 5a with Figure $2 \mathrm{~b}$ shows that the diffraction features from the primary structure are retained, with largely diminished hutshaped wings. However, two main differences are noticed. First, clearly discernible diffraction 
spots with systematic absences are observed at a similar image height (indicated by the arrow in Figure 5a), which are too weak to recognize from a freshly prepared sample (Figure 2c). Second, a few low-intensity streaks coinciding with the horizontal positions of the new spots are discernible, which become clearer at a higher $\theta_{\text {in }}=3.0^{\circ}$ (Figure $5 \mathrm{~b}$ ). As discussed earlier, these new spots and streaks should originate from the orders of the alkyl chains and the sulfur headgroups, respectively, in the emerging structure. These two new diffraction features are also present in patterns recorded at other azimuths (Figure 6, a-d), and their appearance is found to be essentially symmetric with respect to the center streak. We further note that the intensity of the new streaks is much weaker compared to that from the primary structure. The observed new streaks in Figure 5 give a clear indication that the sulfur headgroups of the secondary structure

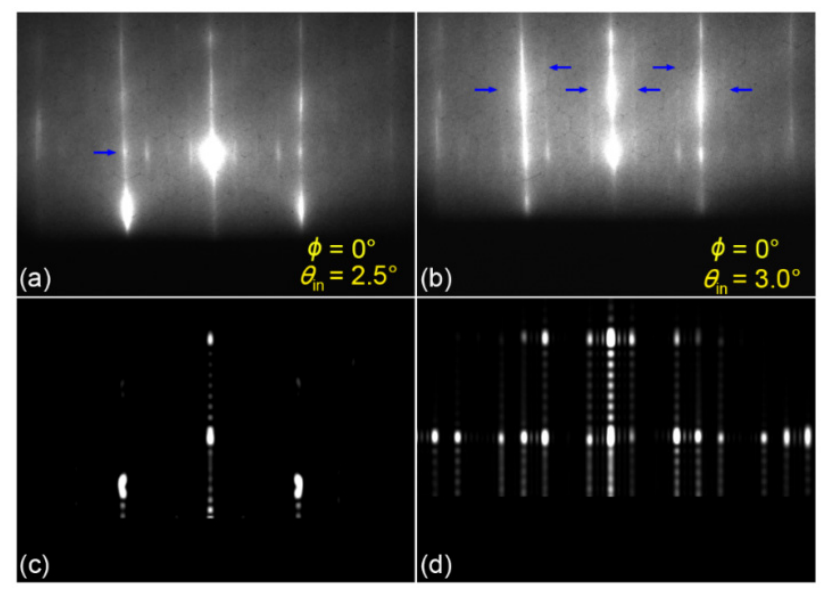

Figure 5. RHEED images of an annealed sample, recorded at $\phi=0^{\circ}$ at $100 \mathrm{~K}$ for two selected incidence angles, (a) $\theta_{\text {in }}=2.5^{\circ}$ and (b) $\theta_{\text {in }}=3.0^{\circ}$. The blue arrows indicate the important diffraction features for structural determination (see text). (c, d) Simulated patterns using the combination of two different models (Figure 1, $\mathrm{c}$ and $\mathrm{g}$ for the sulfur sublattice and Figure $1 \mathrm{~h}$ for the alkyl chains). Four domains whose chain-tilt directions are $60^{\circ}, 120^{\circ}, 240^{\circ}$, and $300^{\circ}$ in azimuth with respect to the electron probe direction are considered in c; the two domains with the tilt directions of $0^{\circ}$ and $180^{\circ}$ (i.e., parallel to the electron incident plane) are used in d. 

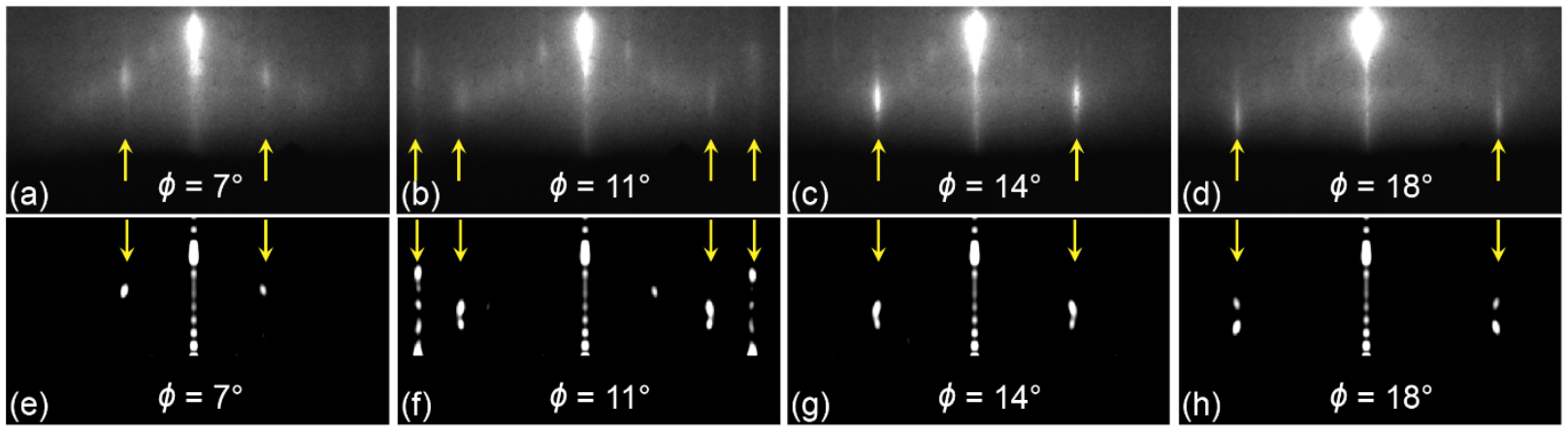

Figure 6. RHEED images of an annealed sample. (a-d) Experimental patterns recorded at selected azimuths. (e-h) Simulated patterns considering all six-fold rotated domains formed by of the two models shown in Figure 1, c, g, and h. The yellow arrows highlight the matching of diffractions seen in the experimental and simulated patterns.

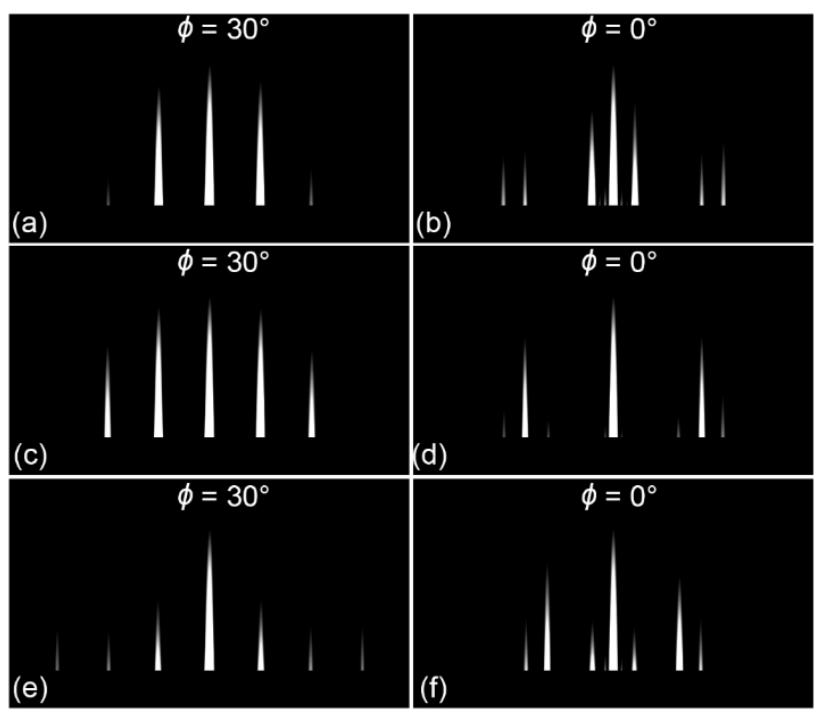

Figure 7. Simulated RHEED patterns for the two principal zones using the $2 \mathrm{D}$ arrangements of sulfur atoms shown in (a, b) Figure 1c, (c, d) Figure 1e, and (e, f) Figure 1g.

no longer maintain the primary hexagonal lattice. Thus, this result does not support the original model assuming equivalent sulfur headgroups by the helium atom scattering ${ }^{20}$ and early $\mathrm{STM}^{29}$ studies (Figure 1, a and d). However, a quick analysis gives support for the $(2 \sqrt{3} \times 3)_{\text {rect }}$ secondary structure. At $\phi=0^{\circ}$, the real-space cell constant associated with the smallest horizontal 
spacing between streaks, $s_{\min }$, is $2 \pi / s_{\min }$ for a rectangular lattice; $s_{\min }$ is a quarter of the spacing $s_{\mathrm{Au}}$ in Figure $2 \mathrm{~d}$ associated with the unit-cell constant of $\mathrm{Au}(111)$, and $a_{\mathrm{Au}}=4 \pi / \sqrt{3} s_{\mathrm{Au}}$. Thus, the cell constant of $2 \pi / s_{\min }=2 \sqrt{3} a_{\mathrm{Au}}=10.0 \AA$ is obtained, matching the first part of the structural assignment. The systematic absences require a certain intracell arrangement of the sulfur atoms.

Shown in Figure 7 are the RHEED simulations for the two high-symmetry zones at $\phi=0^{\circ}$ and $30^{\circ}$ using the different $2 \mathrm{D}$ lattices of sulfur atoms shown in Figure 1. Consistent with the experimental results, the streak patterns at $\phi=30^{\circ}$ do not exhibit a fundamental difference and they are similar to that observed from the primary structure (Figure $2 \mathrm{~g}$ ). However, major changes are seen at $\phi=0^{\circ}$. It is clear that Figure $7 \mathrm{~d}$ fails to produce the superlattice streaks observed in Figure 5b, which signifies a much minor contribution, if ever present, given by the model depicted in Figure 1e. On the other hand, Figure 7, b and f, produces more streaks with the needed absence of the (20) superlattice streak. Given the similar intensity level of the (10) and (30) superlattice streaks as well as the presence of the (50) one in Figure 5b, a combination of Figure $7, b$ and $f$, is therefore needed to be consistent with the observation, instead of a single dominant contributor. Thus, we consider that the best picture for the sulfur sublattice is described by a mixture of the models in Figure 1, c and $\mathrm{g}$. This finding is in fact in an agreement with both of the earlier GIXD studies made by two different groups. ${ }^{21,44}$ Our temperature-dependent results indicate that the S-S dimer formation becomes noticeable at an annealing temperature of $347 \mathrm{~K}$ and above. However, just as a SAM on gold before annealing is a highly inhomogeneous system with multiple contributing species and rotated domains, a mixture of more than one superlattice structure can be anticipated. Here, we also note that both structures exhibit shorter S-S distances (2.2 $\AA$ and $3.2 \AA$, respectively) compared to those in the other models. This tendency of sulfur dimerization upon annealing together with the pairing of alkyl chains and the difference in the 
gold adatom configuration (see below) sheds light on the changes made to a SAM by a thermal treatment.

For the aliphatic chains, a similar examination was conducted using the different arrangements depicted in Figure 1. Judging from the positions where the diffraction spots are observed, the best agreement was found with the model of Figure 1h; the patterns simulated using the combination of the models in Figure 1, c, g, and $\mathrm{h}$ for different azimuths are shown in the bottom panels of Figures 5 and 6 (see also Figure S2 for further comparison). We note that the six-fold azimuthal rotations of domains are still present. In fact, two sets of diffractions can be identified in Figure 5, where the first set (Figure 5c) is generated by those domains with the chain-tilt directions being $60^{\circ}, 120^{\circ}, 240^{\circ}$, and $300^{\circ}$ in azimuth with respect to the electron probe direction, and the second set (Figure 5d) by the domains with the chains parallel to the electron incident plane (i.e., being $0^{\circ}$ and $180^{\circ}$ in azimuth with respect to the electrons' direction). With all six-fold azimuthally rotated domains, diffraction spots away from the center streaks can be reproduced (Figure 6). Some additional features are noticed essentially along the hut-shaped wings, which we attribute to the presence of minor secondary superlattice structures of the alkyl chains resulting from annealing. For example, the two spots near by the center spot in Figure $6 \mathrm{~b}$ may be attributed to the twisted-chain model in Figure 1d (see Figure S2i).

The diffraction consistence with the model of Figure $1 \mathrm{~h}$ indicates that a thermal treatment of a SAM leads to the pairing of aliphatic chains in addition to the aforementioned two types of pairings of sulfur atoms. Evidently, the pairing of whole thiolate molecules, including the headgroups and the aliphatic chains, is responsible for the superlattice structure. These findings provide a consistent picture yet further details compared to previous reports using other methods, e.g., the presence of more than one headgroup structure proposed by the GIXD study ${ }^{44}$ as well as 
the confirmation of both pairings of the sulfur atoms and the chains, which remains a question in the STM study that first resolved a pairing distance of $3.3 \AA .^{30}$ The precise driving force for the pairings is uncertain and may likely be a combination of a few factors, including the enhanced van der Waals interactions between long alkyl chains and an energetic preference resulting in closer S-S association. Here, it is noted that the about-one-order-of-magnitude increase in the average range of ordered domains, from $9.3 \mathrm{~nm}$ as deposited to $87 \mathrm{~nm}$ after annealing, demonstrates the importance of domain sizes to the formation of the superlattice, which is in an agreement with the literature. $^{32}$

\subsection{Gold adatoms underneath thiolates}

Here, we address the issue regarding the presence of gold adatoms below the sulfur headgroups. The experimental RHEED images show that the diffraction streaks from the sulfur sublattice of the $(2 \sqrt{3} \times 3)_{\text {rect }}$ structure (Figure 5 b) are much weaker in intensity compared to those from the $(\sqrt{3} \times \sqrt{3}) R 30^{\circ}$ primary structure (Figure 2$)$. Given the same scattering cross section of electrons for sulfur atoms, two scenarios are possible for such a difference. The first is related to the population difference, i.e., the proportion of the superlattice structure might still be in a lesser extent and not as high as that of the primary structure even as the ordered domains are enlarged after a high-temperature annealing. Although such a scenario cannot be completely ruled out, it does not resolve a major inconsistency. As anticipated in the kinematic scattering theory, the simulated RHEED patterns yield comparable streak intensities (but with substantial differences in the positions) from the sulfur sublattices of the two structures. However, a large intensity discrepancy is found between the experimental and simulated images for the primary structure before annealing. This leads us to consider the second scenario with the presence of gold 


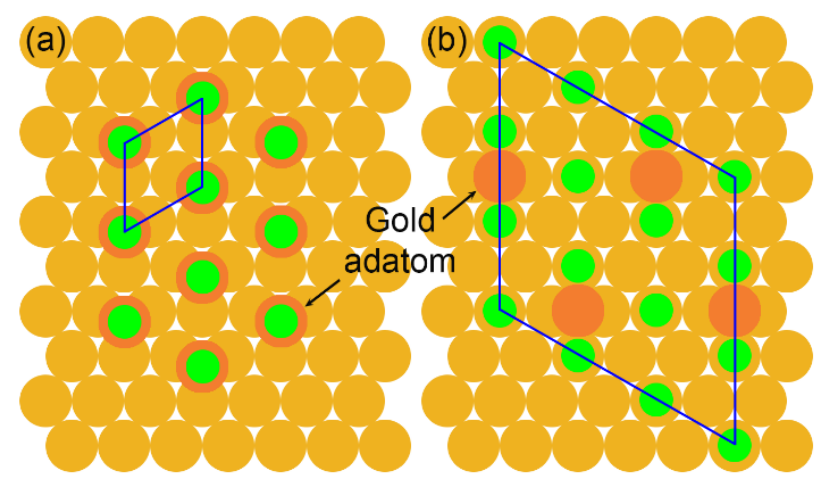

Figure 8. Schematic of two adatom models for the primary structure, with (a) (RS-Au $\left.u_{a d}\right)$ units and (b) a supercell structure proposed recently in Ref. 59.

adatoms, which have a substantially higher scattering capability compared to sulfur atoms in the primary structure.

The experimentally observed streaks (Figure 2) require the same unit cell symmetry for the gold adatoms as the hexagonal arrangement of the sulfur headgroups. Shown in Figure 8 are two candidates suggested by different studies. The first model is atop site binding of the thiolates to gold adatom $\left(\mathrm{RS}-\mathrm{Au}_{\mathrm{ad}}\right)$ based on normal incidence $\mathrm{x}$-ray standing waves $(\mathrm{NIXW})^{56}$ and photoemission core-level shifts ${ }^{57}$ for short-chain alkanethiols (Figure 8a). For this $(\sqrt{3} \times$ $\sqrt{3}) R 30^{\circ}$ primary structure, the surface coverage of gold adatoms is 0.33 referenced to $\mathrm{Au}(111)$, i.e. one gold adatom per SAM unit cell that is the same as the thiolate coverage. The second model contains gold adatom-dithiolate $\left(\mathrm{RS}-\mathrm{Au}_{\mathrm{ad}}-\mathrm{SR}\right)$ staple motifs, which have been observed for short-chain alkanethiols on planar gold surfaces ${ }^{45}$ as well as on gold nanoparticles. ${ }^{58}$ Construction of a $(\sqrt{3} \times \sqrt{3}) R 30^{\circ}$ lattice using the RS-Au $\mathrm{ad}^{-} \mathrm{SR}$ motifs is not straightforward, although they have been used to construct other types of unit cells observed in SAMs. ${ }^{35}$ Here, the recent model proposed by Torrelles et al. based on GIXD and STM data is considered, which contain both dithiolate staple units and thiolate radicals in a $(3 \sqrt{3} \times 3 \sqrt{3}) R 30^{\circ}$ supercell structure 
with one third of the coverage in the first model (Figure 8b). ${ }^{59}$

Hence, we compare the simulated patterns at $\phi=30^{\circ}$ and $\theta_{\text {in }}=1.3^{\circ}$ for the different models (Figure 9) with the experimental RHEED image in Figure 2f. It is found that compared to the aliphatic chain Bragg spots, the diffraction streaks are discernable only when gold adatoms are bonded to all thiolates (Figure 9a). The low streak intensity in Figure 9b signifies that a lower coverage of gold adatoms fails to provide an effective scattering contribution, which results in a pattern resembling that for the original model without gold adatoms (Figure 9c) and thus may be ruled out. Thus, our study suggests that the primary structure consists of gold-adatom-bonded monothiolates as the basic structural moiety. On the other hand, for the $(2 \sqrt{3} \times 3)_{\text {rect }}$ superlattice structure, we are led to conclude that the coverage of gold adatoms, if ever present, would be significantly lower than that for the primary structure or even of minor importance because of the low streak intensity compared to that of the diffraction spots from the reconstructed alkyl chains.

Thus, the significant difference in the coverage of gold adatoms between the primary and superlattice structures is worth further investigation, taking into account the influence of alkyl chain lengths (and hence the strength of van der Waals interactions), fabrication and thermal treatment processes, and metal surface conditions. It is noted that a low coverage $(0.14-0.17)$ of gold adatoms associated with a dithiolate-bonded model has been reported in previous STM studies, ${ }^{60,61}$ although the results were obtained and interpreted using irreversible chemical changes. As a further confirmation, we performed another simulation (not shown) with a $(2 \sqrt{3} \times 2 \sqrt{3}) R 30^{\circ}$ supercell whose coverage is 0.16 (i.e., $2: 1$ for the ratio between sulfur and gold atoms for dithiolate staple motifs). The resulting diffraction pattern shows not only much weaker streak intensities but additional streaks in between that do not conform with the symmetry of the observed $(\sqrt{3} \times \sqrt{3}) R 30^{\circ}$ lattice. 


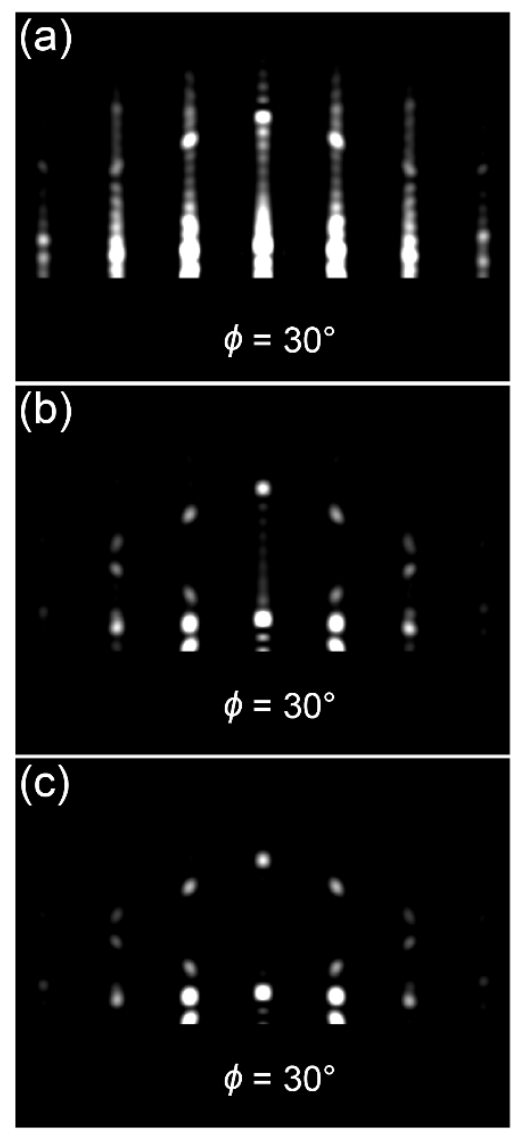

Figure 9. Simulated RHEED patterns at $\phi=30^{\circ}$ for the six-fold azimuthally rotated, $(\sqrt{3} \times$ $\sqrt{3}) R 30^{\circ}$ primary structure, with $(\mathrm{a})\left(\mathrm{RS}-\mathrm{Au}_{\mathrm{ad}}\right)$ units shown in Figure $8 \mathrm{a}$, (b) the model proposed in Ref. 59 as shown in Figure 8b, and (c) without gold adatoms below the sulfur headgroups.

Thus, by measuring SAMs in their original film conditions, we have found evidence that a high coverage of gold adatoms as in the monothiolate-bonded model is needed, which has also been argued and considered by Li et al. using STM results. ${ }^{62}$ On the contrary, the high coverage of gold adatoms is lost in the $(2 \sqrt{3} \times 3)_{\text {rect }}$ superlattice structure formed via annealing. This observation becomes reasonable and may be anticipated considering the observation of enlarged domains of long aliphatic chains and closer S-S distances in the headgroups. Through thermal annealing, a better methylene packing takes place during the structural transition as a result of the strong van der Waals interactions between long chains. However, the unit cell structure of 
Figure $1 \mathrm{~h}$ is not compatible with the headgroups and gold adatoms in the original primary lattice. Hence, we find support that the headgroups favor closer S-S distances without bonding to gold adatoms, resulting in a significant reduction in the coverage. The two models of Figure 1, c and g, especially the latter, may indicate the presence of some gold adatoms. The unbonded atoms may migrate to the step edges and vacancy islands, as previously observed during the desorption of thiolates. ${ }^{62,63}$

\section{Conclusions}

The structures of self-assembled monolayers of octadecanethiols chemisorbed on singlecrystalline $\mathrm{Au}(111)$ were investigated using RHEED and kinematic scattering theory simulations. The as-prepared films, even after an extended deposition time, contain small ordered domains of a primary $(\sqrt{3} \times \sqrt{3}) R 30^{\circ}$ structure with a molecular tilt of $30.2^{\circ}$ and six-fold azimuthal rotation for the tilt, along with azimuthally disorderly packed regions of the same average tilt. Gold adatoms with the same hexagonal symmetry below the sulfur headgroups are present in the primary structure to account for the large intensity of the diffraction streaks; such a high coverage of gold adatoms is needed. The annealing of the films up to a temperature of $383 \mathrm{~K}$ results in a significant portion of the ordered domains transitioning to the $(2 \sqrt{3} \times 3)_{\text {rect }}$ superlattice structure, with an increase in the average size from 9.3 to $87 \mathrm{~nm}$, for both the sulfur atoms and the alkyl chains; the disorderly packed areas are largely reduced. Upon further examinations, the superlattice structure consists of a mixture of two sulfur arrangements, both of which contain a short S-S distance, and the pairwise-packed aliphatic chains, without the presence of gold adatoms. Concurrently, the coverage of gold adatoms is largely reduced, as evidenced in the much weaker streak diffractions observed. A microscopic model consistent with 
the changes in the structures, cell packings, headgroup distances, and coverages of gold adatoms is provided. From these results, we believe that RHEED is a helpful method to further resolve structural details and questions about other self-assembled films, as demonstrated in this study which contain two or more regions of sublattices.

\section{Conflicts of interest}

The authors declare no competing financial interests.

\section{- Acknowledgements}

This research was supported by a National Science Foundation CAREER Award (Grant no. CHE-1653903) and the R. A. Welch Foundation (Grant no. E-1860).

\section{Footnotes}

\section{Electronic Supporting Information}

Schematic of a typical RHEED set-up and comparison of simulated RHEED patterns using different superlattice structures for the aliphatic chains

\section{- AUTHOR INFORMATION}

\section{Corresponding Author}

*E-mail: yang@uh.edu Phone: +1 713-743-6022.

\section{ORCID}

Ding-Shyue Yang: 0000-0003-2713-9128

\section{References}

1 R. G. Nuzzo and D. L. Allara, Adsorption of Bifunctional Organic Disulfides on Gold Surfaces., J. Am. Chem. Soc., 1983, 105, 4481-4483. 

Sensor Arrays., Acc. Chem. Res., 1998, 31, 219-227.

3 Li Deng, A. Milan Mrksich and G. M. Whitesides, Self-Assembled Monolayers of Alkanethiolates Presenting Tri(Propylene Sulfoxide) Groups Resist the Adsorption of Protein., J. Am. Chem. Soc., 1996, 118, 5136-5137.

4 M. Mrksich, C. S. Chen, Y. Xia, L. E. Dike, D. E. Ingber and G. M. Whitesides, Controlling Cell Attachment on Contoured Surfaces with Self-Assembled Monolayers of Alkanethiolates on Gold., Proc. Natl. Acad. Sci. U. S. A., 1996, 93, 10775-8.

5 S. Flink, F. C. J. M. Van Veggel and D. N. Reinhoudt, Sensor Functionalities in SelfAssembled Monolayers., Adv. Mater., 2000, 12, 1315-1328.

6 I. Willner and E. Katz, Integration of Layered Redox Proteins and Conductive Supports for Bioelectronic Applications., Angew. Chem. Int. Ed., 2000, 39, 1180-1218.

7 J. M. Tour, Molecular Electronics. Synthesis and Testing of Components., Acc. Chem. Res., 2000, 33, 791-804.

8 C. Joachim, J. K. Gimzewski and A. Aviram, Electronics Using Hybrid-Molecular and Mono-Molecular Devices., Nature, 2000, 408, 541-548.

9 A. R. Pease, J. O. Jeppesen, J. F. Stoddart, Y. Luo, C. P. Collier and J. R. Heath, Switching Devices Based on Interlocked Molecules., Acc. Chem. Res., 2001, 34, 433-444.

10 R. Qiao and L. Zuo, Self-Assembly Monolayers Boosting Organic-Inorganic Halide Perovskite Solar Cell Performance., J. Mater. Res., 2018, 33, 387-400.

11 S. Q. Lud, M. Steenackers, R. Jordan, P. Bruno, D. M. Gruen, P. Feulner, J. A. Garrido and M. Stutzmann, Chemical Grafting of Biphenyl Self-Assembled Monolayers on Ultrananocrystalline Diamond., J. Am. Chem. Soc., 2006, 128, 16884-16891.

12 D. Liu and Q. Miao, Recent Progress in Interface Engineering of Organic Thin Film Transistors with Self-Assembled Monolayers., Mater. Chem. Front., 2018, 2, 11-21.

13 K. Tamada, M. Hara, H. Sasabe and W. Knoll, Surface Phase Behavior of N-Alkanethiol Self-Assembled Monolayers Adsorbed on Au(111): An Atomic Force Microscope Study., Langmuir, 1997, 13, 1558-1566.

14 S. Xu, S. J. N. Cruchon-Dupeyrat, J. C. Garno, G. Y. Liu, G. K. Jennings, T. H. Yong and P. E. Laibinis, In Situ Studies of Thiol Self-Assembly on Gold from Solution Using Atomic Force Microscopy., J. Chem. Phys., 1998, 108, 5002-5012.

15 G. E. Poirier, Mechanism of Formation of Au Vacancy Islands in Alkanethiol Monolayers on $\mathrm{Au}(111)$., Langmuir, 1997, 13, 2019-2026.

16 G. E. Poirier and E. D. Pylant, The Self-Assembly Mechanism of Alkanethiols on $\mathrm{Au}(111) .$, Science, 1996, 272, 1145-1148.

17 L. H. Dubois, B. R. Zegarski and R. G. Nuzzo, Molecular Ordering of Organosulfur Compounds on $\mathrm{Au}(111)$ and $\mathrm{Au}(100)$ : Adsorption from Solution and in Ultrahigh Vacuum., J. Chem. Phys., 1993, 98, 678-688. 
18 M. D. Porter, T. B. Bright, D. L. Allara and C. E. D. Chidsey, Spontaneously Organized Molecular Assemblies. 4. Structural Characterization of n-Alkyl Thiol Monolayers on Gold by Optical Ellipsometry, Infrared Spectroscopy, and Electrochemistry., J. Am. Chem. Soc., 1987, 109, 3559-3568.

19 R. G. Nuzzo, B. R. Zegarski and L. H. DuBois, Fundamental Studies of the Chemisorption of Organosulfur Compounds on $\mathrm{Au}(111)$. Implications for Molecular Self-Assembly on Gold Surfaces., J. Am. Chem. Soc., 1987, 109, 733-740.

20 N. Camillone, C. E. D. Chidsey, G. Y. Liu and G. Scoles, Superlattice Structure at the Surface of a Monolayer of Octadecanethiol Self-Assembled on Au(111)., J. Chem. Phys., 1993, 98, 3503-3511.

21 P. Fenter, A. Eberhardt and P. Eisenberger, Self-Assembly of n-Alkyl Thiols as Disulfides on $\mathrm{Au}(111)$., Science, 1994, 266, 1216-1218.

22 C. Vericat, M. E. Vela, G. Benitez, P. Carro and R. C. Salvarezza, Self-Assembled Monolayers of Thiols and Dithiols on Gold: New Challenges for a Well-Known System., Chem. Soc. Rev., 2010, 39, 1805-1834.

23 D. P. Woodruff, The Interface Structure of N-Alkylthiolate Self-Assembled Monolayers on Coinage Metal Surfaces., Phys. Chem. Chem. Phys., 2008, 10, 7211-7221.

24 C. E. D. Chidsey, G. Y. Liu, P. Rowntree and G. Scoles, Molecular Order at the Surface of an Organic Monolayer Studied by Low Energy Helium Diffraction., J. Chem. Phys., 1989, 91, 4421-4423.

25 L. Strong and G. M. Whitesides, Structures of Self-Assembled Monolayer Films of Organosulfur Compounds Adsorbed on Gold Single Crystals: Electron Diffraction Studies., Langmuir, 1988, 4, 546-558.

26 C. E. D. Chidsey and D. N. Loiacono, Chemical Functionality in Self-Assembled Monolayers: Structural and Electrochemical Properties., Langmuir, 1990, 6, 682-691.

27 R. G. Nuzzo, E. M. Korenic and L. H. Dubois, Studies of the Temperature-dependent Phase Behavior of Long Chain $n$-alkyl Thiol Monolayers on Gold., J. Chem. Phys., 1990, 93, 767-773.

28 L. Müller-Meskamp, B. Lüssem, S. Karthäuser and R. Waser, Rectangular $(3 \times 2 \sqrt{ } 3)$ Superlattice of a Dodecanethiol Self-Assembled Monolayer on $\mathrm{Au}(111)$ Observed by Ultra-High-Vacuum Scanning Tunneling Microscopy., J. Phys. Chem. B, 2005, 109, 11424-11426.

29 G. E. Poirier and M. J. Tarlov, The c(4×2) Superlattice of n-Alkanethiol Monolayers SelfAssembled on Au(111)., Langmuir, 1994, 10, 2853-2856.

30 F. Li, L. Tang, W. Zhou and Q. Guo, Relationship between the $c(4 \times 2)$ and the $(\sqrt{3} \times \sqrt{ } 3)$ R30 ${ }^{\circ}$ Phases in Alkanethiol Self-Assembled Monolayers on Au(111)., Phys. Chem. Chem. Phys., 2011, 13, 11958-11964.

31 I. Touzov and C. B. Gorman, Tip-Induced Structural Rearrangements of Alkanethiolate Self-Assembled Monolayers on Gold., J. Phys. Chem. B, 2002, 101, 5263-5276. 
32 E. Paradis and P. Rowntree, The Structure and Evolution of Self-Assembled Butanethiol Films on Gold Substrates: Part I: STM Investigations of the c $(4 \times 2)$ and Striped Phases., $J$. Electroanal. Chem., 2003, 550-551, 175-185.

33 F. Schreiber, Structure and Growth of Self-Assembling Monolayers., Prog. Surf. Sci., 2000, 65, 151-257.

34 L. Zhang, W. A. Goddard and S. Jiang, Molecular Simulation Study of the $\mathrm{c}(4 \times 2)$ Superlattice Structure of Alkanethiol Self-Assembled Monolayers on Au(111)., J. Chem. Phys., 2002, 117, 7342-7349.

35 Q. Guo and F. Li, Self-Assembled Alkanethiol Monolayers on Gold Surfaces: Resolving the Complex Structure at the Interface by STM., Phys. Chem. Chem. Phys., 2014, 16, 19074-19090.

36 A. Chaudhuri, T. J. Lerotholi, D. C. Jackson, D. P. Woodruff and R. G. Jones, $(2 \sqrt{3} \times 3)$ Rect.Phase of Alkylthiolate Self-Assembled Monolayers on $\mathrm{Au}(111)$ : A SymmetryConstrained Structural Solution., Phys. Rev. B - Condens. Matter Mater. Phys., 2009, 79, 195439 .

37 M. S. Yeganeh, S. M. Dougal, R. S. Polizzotti and P. Rabinowitz, Interfacial Atomic Structure of a Self-Assembled Alkyl Thiol Monolayer/Au(111): A Sum-Frequency Generation Study., Phys. Rev. Lett., 1995, 74, 1811-1814.

38 C. Zubr, C. Deuper, F. Schneider, M.Neumann, M.Grunze and A.Schertel, The presence of two different sulfur species in self-assembled films of n-alkanethiols on $\mathrm{Au}$ and $\mathrm{Ag}$ surfaces, Chem. Phys. Lett., 1995, 238, 308-312.

39 N. Nishida, M. Hara, H. Sasabe and W. Knoll, Dimerization Process in Alkanethiol SelfAssembled Monolayer on Au(111)., Japanese J. Appl. Physics, Part 2 Lett., 1996, 35, L799-L802.

40 J. Voets, J. W. Gerritsen, R. F. P. Grimbergen and H. Van Kempen, Chain-LengthDependent Structure of Alkanethiols Forming Dimers on Au(111)., Surf. Sci., 1998, 399, 316-323.

41 P. Fenter, F. Schreiber, L. Berman, G. Scoles, P. Eisenberger and M. J. Bedzyk, On the Structure and Evolution of the Buried S/Au Interface in Self-Assembled Monolayers: XRay Standing Wave Results., Surf. Sci., 1998, 412-413, 213-235.

42 G. J. Kluth, C. Carraro and R. Maboudian, Direct Observation of Sulfur Dimers in Alkanethiol Self-Assembled Monolayers on Au(111)., Phys. Rev. B, 1999, 59, R10449R10452.

43 M. Wirde, U. Gelius, T. Dunbar and D. L. Allara, Modification of Self-Assembled Monolayers of Alkanethiols on Gold by Ionizing Radiation., Nucl. Instruments Methods Phys. Res. Sect. B Beam Interact. with Mater. Atoms, 1997, 131, 245-251.

44 X. Torrelles, E. Barrena, C. Munuera, J. Rius, S. Ferrer and C. Ocal, New Insights in the $\mathrm{c}(4 \times 2)$ Reconstruction of Hexadecanethiol on $\mathrm{Au}(111)$ Revealed by Grazing Incidence XRay Diffraction., Langmuir, 2004, 20, 9396-9402. 
45 P. Maksymovych, D. C. Sorescu and J. T. Yates, Gold-Adatom-Mediated Bonding in Self-Assembled Short-Chain Alkanethiolate Species on the Au(111) Surface., Phys. Rev. Lett., 2006, 97, 146103.

46 C. Vericat, G. Andreasen and M. E. Vela, Following Transformation in Self-Assembled Alkanethiol Monolayers on $\mathrm{Au}(111)$ by in Situ Scanning Tunneling Microscopy., J. Chem. Phys., 2001, 115, 6672-6678.

47 G. J. Russell, M. C. Petty, I. R. Peterson, G. G. Roberts, J. P. Lloyd and K. K. Kan, RHEED Study of Cadmium Stearate Langmuir-Blodgett Films., J. Mater. Sci. Lett., 1984, 3, 25-28.

48 I. R. Peterson, G. J. Russell, J. D. Earls and I. R. Girling, Surface Pressure Dependence of Molecular Tilt in Langmuir-Blodgett Films of 22-Tricosenoic Acid., Thin Solid Films, 1988, 161, 325-331.

49 I. Robinson, J. R. Sambles and I. R. Peterson, Reflection High Energy Electron Diffraction Analysis of the Orientation of the Monoclinic Subcell of 22-Tricosenoic Acid Langmuir-Blodgett Bilayers as a Function of the Deposition Pressure., Thin Solid Films, 1989, 172, 149-158.

50 V. Brzezinski and I. R. Peterson, Deposition Effects on the Structure of LangmuirBlodgett Monolayers of Docosanoic Acid., J. Phys. Chem., 1995, 99, 12545-12552.

51 R. G. Nuzzo, L. H. Dubois and D. L. Allara, Fundamental Studies of Microscopic Wetting on Organic Surfaces. 1. Formation and Structural Characterization of a Self-Consistent Series of Polyfunctional Organic Monolayers., J. Am. Chem. Soc., 1990, 112, 558-569.

52 B. E. Warren, X-ray diffraction, Dover Publications, 1990.

53 J. Zhang, Q. Chi and J. Ulstrup, Assembly Dynamics and Detailed Structure of 1Propanethiol Monolayers on $\mathrm{Au}(111)$ Surfaces Observed Real Time by in Situ STM., Langmuir, 2006, 22, 6203-6213.

54 J. C. Love, L. A. Estroff, J. K. Kriebel, R. G. Nuzzo and G. M. Whitesides, SelfAssembled Monolayers of Thiolates on Metals as a Form of Nanotechnology., Chem. Rev., 2005, 105, 1103-1170.

55 T. Y. B. Leung, M. C. Gerstenberg, D. J. Lavrich, G. Scoles, F. Schreiber and G. E. Poirier, 1,6-Hexanedithiol Monolayers on $\mathrm{Au}(111)$ : A Multitechnique Structural Study., Langmuir, 2000, 16, 549-561.

56 M. Yu, N. Bovet, C. J. Satterley, S. Bengió, K. R. J. Lovelock, P. K. Milligan, R. G. Jones, D. P. Woodruff and V. Dhanak, True Nature of an Archetypal Self-Assembly System: Mobile Au-Thiolate Species on Au(111)., Phys. Rev. Lett., 2006, 97, 166102.

57 A. Chaudhuri, T. J. Lerotholi, D. C. Jackson, D. P. Woodruff and V. Dhanak, Local Methylthiolate Adsorption Geometry on $\mathrm{Au}(111)$ from Photoemission Core-Level Shifts., Phys. Rev. Lett., 2009, 102, 126101.

58 H. Häkkinen, The Gold-Sulfur Interface at the Nanoscale., Nat. Chem., 2012, 4, 443-455. 
59 X. Torrelles, E. Pensa, E. Cortés, R. Salvarezza, P. Carro, C. Hernández Guerrero, C. Ocal, E. Barrena and S. Ferrer, Solving the Long-Standing Controversy of Long-Chain Alkanethiols Surface Structure on Au(111)., J. Phys. Chem. C, 2018, 122, 3893-3902.

60 N. A. Kautz and S. A. Kandel, Alkanethiol/Au(111) Self-Assembled Monolayers Contain Gold Adatoms: Scanning Tunneling Microscopy before and after Reaction with Atomic Hydrogen., J. Am. Chem. Soc., 2008, 130, 6908-6909.

61 N. A. Kautz and S. A. Kandel, Alkanethiol Monolayers Contain Gold Adatoms, and Adatom Coverage Is Independent of Chain Length., J. Phys. Chem. C, 2009, 113, 1928619291.

62 F. Sen Li, W. Zhou and Q. Guo, Uncovering the Hidden Gold Atoms in a Self-Assembled Monolayer of Alkanethiol Molecules on Au(111)., Phys. Rev. B - Condens. Matter Mater. Phys., 2009, 79, 1-4.

63 Q. Guo, X. Sun and R. E. Palmer, Structural Dynamics Induced by Self-Assembled Monolayers on Au(111)., Phys. Rev. B - Condens. Matter Mater. Phys., 2005, 71, 1-5. 


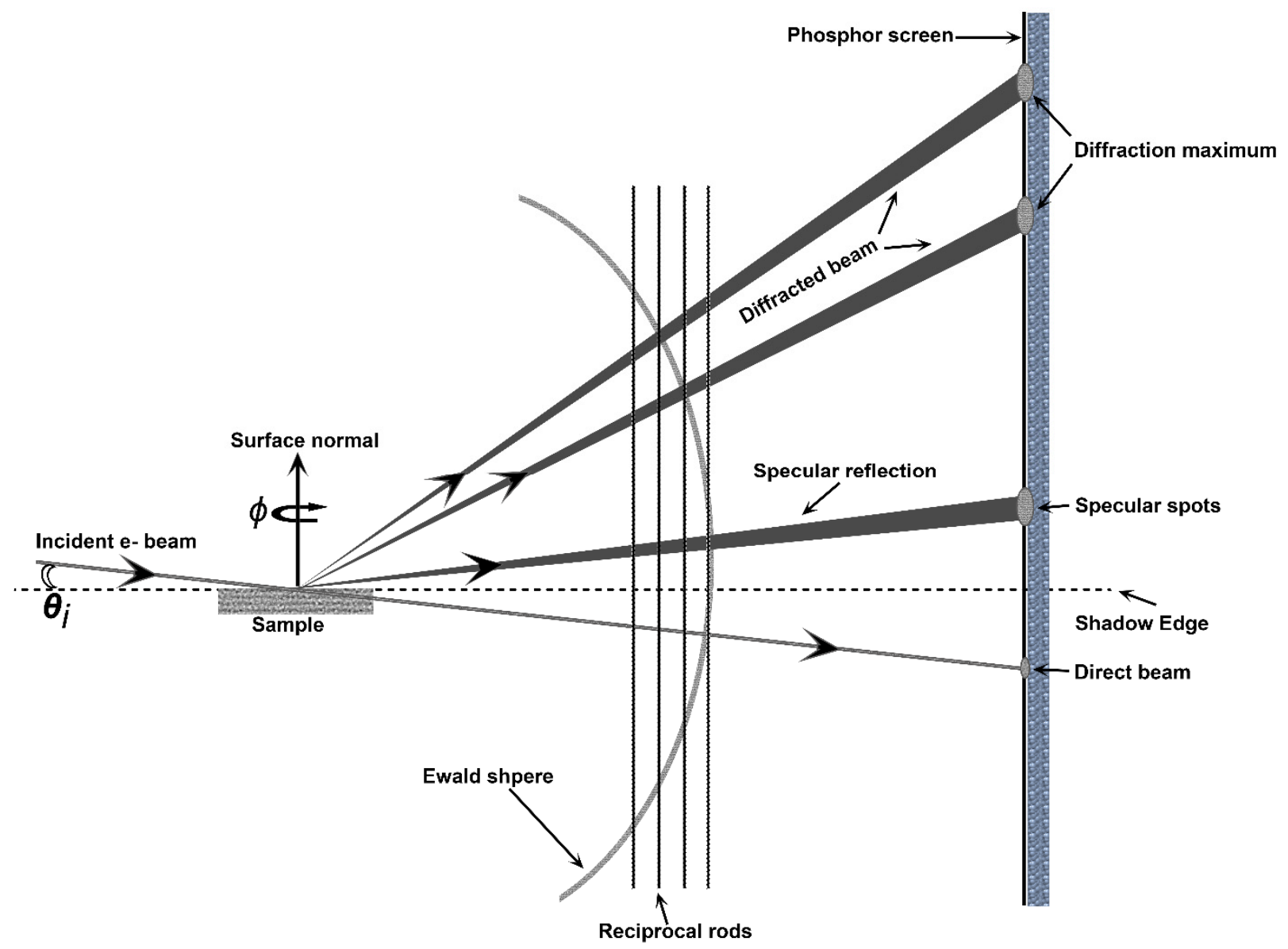

Figure S1. Schematic of a RHEED set-up and the Ewald construction for image formation from a two-dimensional lattice. 


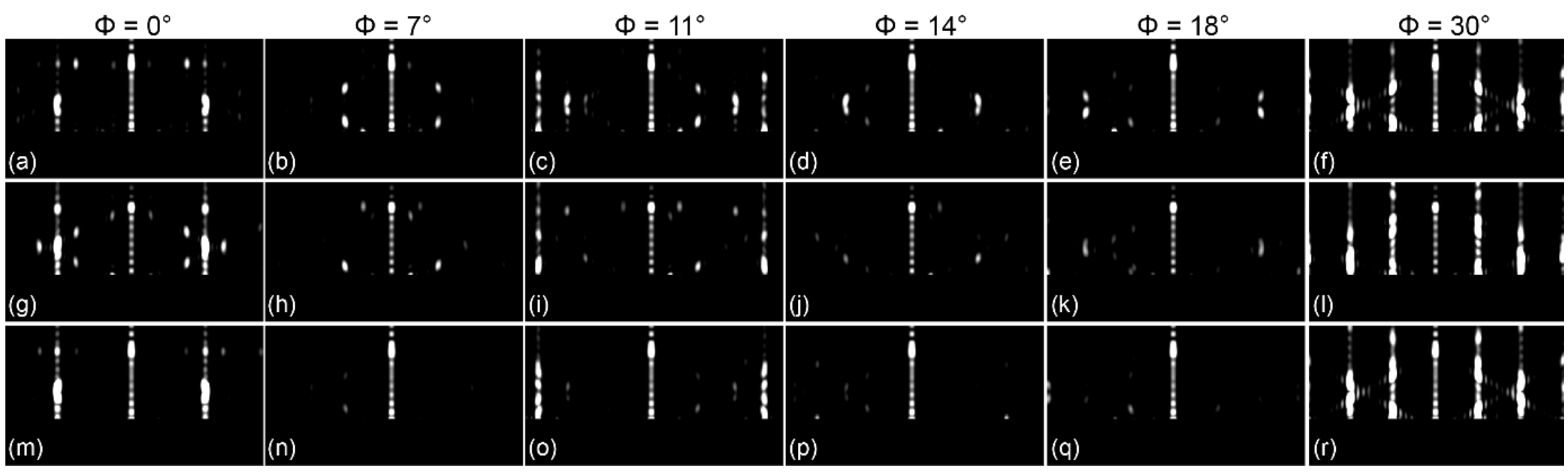

Figure S2. Simulated RHEED patterns according to three $(2 \sqrt{3} \times 3)_{\text {rect }}$ structures for select azimuths. $(\mathrm{a}-\mathrm{f})$ Based on Figure $1 \mathrm{~h}$ with a combination of the models shown in Figure 1, $\mathrm{c}$ and $\mathrm{g}$ for the sulfur headgroups. (g-1) Based on Figure 1d with the sulfur headgroups as in Figure 1a. (m-r) Based on Figure $1 \mathrm{f}$ with the sulfur headgroups as in Figure 1e. 